\title{
Topology research of deexcitation system for large generator based on soft-switching technology
}

\author{
Qinyue Tan ${ }^{1, a}$, Jianhang Zhu ${ }^{2}$, Shiying Wang ${ }^{3}$, Sihang Cui ${ }^{1}$, Lei Liang ${ }^{1}$
}

1 Department of Power and Electrical Engineering, College of Water Resources and Architectural Engineering, Northwest A\&F University, Yang Ling, Shaanxi Province, China 712100

2 School of Electrical and Electronic Engineering, Huazhong University of Science and Technology, Wu Han, Hubei, P. R. China 430074

3 School of Electrical Engineering, Chongqing University, Chongqing, P. R. China 400044

aemail: qinyuetan@126.com

\begin{abstract}
Keywords: large generators; deexcitation; soft-switching; "parallel charged, series discharged"; nonlinear resistor.

Abstract.The damage of overvoltage and arc caused by strongly pulling out the magnetic switching in the traditional process of deexcitation makes the deexcitation problem of the large generator become an important research subject. The technology of power electronics soft-switching provides a new way for the deexcitation of the large generator. In this paper, the topology of deexcitation system and its corresponding control circuit is designed, and it depend on the excitation parameters of the "Three Gorges" power plant. It propose a new way to design the non-linear resistor of energy consumption in deexcitation, or "parallel charged, series discharged", which significantly reduces the impact of voltage in deexcitation process, the deexcitation main switch is turned off when the main circuit current reduce to zero. The theoretical analysis prove that the deexcitation time is $0.51 \mathrm{~s}$ and it's $0.4 \mathrm{~s}$ from the simulation analysis. The results indicate that the deexcitation system with new topology can be fast, safe and reliable.
\end{abstract}

\section{Introduction}

The rated excitation voltage and current of large generator is huge, which give the fast and safe deexcitation a great challenge to synchronous generators. And the current study is more concentrated in the improvement of traditional ways of deexcitation and manufacturing processes of deexcitation equipment. But it cannot meet the demand of deexcitation of large generators, and how to be more safe and rapid for deexcitation is still a problem of large generator. So far, most of the generator excitation apply circuit breaker in series with the excitation DC circuit. With the increase of generator capacity, corresponding parameters of exciter are also increased, and the DC deexcitation cannot meet the need of development of deexcitation technology, and then AC deexcitation system is proposed, and apply circuit breaker in series with the excitation AC side[1-2]. Whether it is a DC or AC deexcitation technology, the success of deexcitation mainly depends on the strength of the excitation switch, including the ability to build voltage of excitation switch, arcing strength and breaking capacity[3]. The electrical intensity of switch arcing is proportional to UI, when the switch arcing energy is greater than the rated capacity of the switch, the switch will cause damage, and it can not succeed implement deexcitation safe and fast, which will result in deexcitation failed and generator crisis[4]. Abroad widely apply matching $\mathrm{SiC}$ nonlinear resistor or linear resistance for deexcitation, and the requirement of switch interdiction strength decreased, which help the switch build voltage and exchange current, but it can only guarantee general rated parameters deexcitation, which don't have protection capability for the ultimate situation [3].

Compare with the traditional deexcitation effects and techniques, this paper proposes a novel soft-switching deexcitation program and its "parallel charged, series discharged" way to build voltage for deexcitation, which make the non-linear resistance start and deexcitation energy consumption quickly. through the theory and simulation analysis, the deexcitation system can be proved fast, secure and reliable. 


\section{The principle of new topology for deexcitation}

This paper presents a design of deexcitation system based on soft-switching technology, as shown in Fig.1, and proposes a new way to build voltage . According to its design features, namely "parallel charged, series discharged", and the system can adapt to different size of the generator excitation system[5].

As shown in Fig. 1, $\mathrm{C}_{1}$ and $\mathrm{C}_{2}$ are the main charging capacitor for deexcitation, and as a filter at the same time in normal operation of excitation system. The linear resistor of $R_{1}, R_{2}$ and $R_{3}$ consume part of magnetic energy at the process of deexcitation. The control of full-controlled devices of $\mathrm{GTO}_{1}$ and $\mathrm{GTO}_{2}$ are depend on the protective relaying signals and the signals of building voltage size, which will create the condition of soft-switching. The nonlinear resistor of $\mathrm{ZnO}$ is the main energy dissipation original $\mathrm{R}$. A inductance $\mathrm{L}$ and a resistor $\mathrm{r}$ series connection, which are equivalent to the excitation winding.

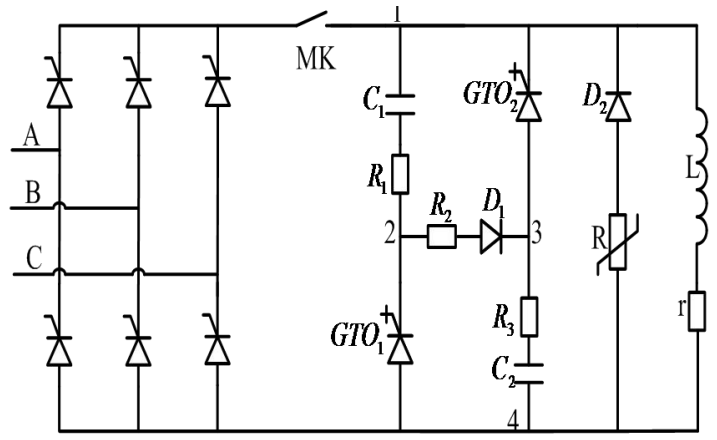

Fig.1 The schematic of new de-excitation system

The active deexcitation capacity make it more suitable for different requirements of different generators, when the generator need to stop, the excitation system should deexcitation. The faults protector of generator or other instructions issue the deexcitation operation command. At the first, turn off the trigger of thyristor rectifier, and make the full-controlled devices $\mathrm{GTO}_{1}$ and $\mathrm{GTO}_{2}$ conduct, so that the main circuit current of excitation is transferred to the new deexcitation system. In this process, capacitors charged by the high-frequency harmonics begin to discharge, forcing the main circuit current of excitation decreases. The main circuit current of excitation drops to zero under the dual role of both, then open the excitation main switch MK. It achieve the effect of zerocurrent-switching for main switch of excitation, and the three-phase sources separate with the excitation system, which is benefit to implement rapid deexcitation.

After these, the energy in field winding began to reverse charge two shunt capacitance. The voltage transformer supervise the voltage $U_{12}$ between 1 and 2 points and voltage $U_{34}$ between 3 and 4 points, and the sum of both $U_{12}$ and $U_{34}$ act as logical switch signal of full-controlled devices both $\mathrm{GTO}_{1}$ and $\mathrm{GTO}_{2}$, when the build voltage reaches the starting value of nonlinear resistance, turn off full-controlled devices both $\mathrm{GTO}_{1}$ and $\mathrm{GTO}_{2}$, then two capacitors of $\mathrm{C}_{1}$ and $\mathrm{C}_{2}$ are connected in series, and their voltage are added, which sufficiently form the high voltage to start non-linear resistor, and improve the conduction condition. The build voltage process is called "parallel charged, series discharged". Finally, the magnetic field energy is consumed by the nonlinear $\mathrm{ZnO}$ resistor and the other three linear resistors.

\section{The theoretical analysis of new topology for deexcitation}

As shown in Fig. 1, original parameter of the new deexcitation system is set up as follows. Two main charging capacitance both $C_{1}$ and $C_{2}$ is $1000 \mu \mathrm{F}$. The resistance both $R_{1}$ and $R_{3}$ is $0.5 \Omega, R_{2}$ is $1 \Omega$. The inductance $\mathrm{L}$ of field winding is $0.3 \mathrm{H}$. The resistor $\mathrm{r}$ of it is $0.11 \Omega$. The resistor $\mathrm{R}$ is nonlinear resistance of $\mathrm{ZnO}$, and its starting voltage is $2300 \mathrm{~V}$. The capacity of three gorges power plant is $700 \mathrm{MW}$, and its excitation current exceed $4 \mathrm{kA}$. The deexcitation residual voltage of $\mathrm{ZnO}$ 
$\mathrm{U}_{\mathrm{R}}=2.2 \mathrm{kV}$, and over-voltage of no-load strong excitation in error reach to 1.3 times of rated value [5]. The corresponding strong excitation voltage is about $\mathrm{U}_{0}=2.2 \mathrm{kV}(1243 \times \sqrt{ } 2 \times 1.3=2262)$.

$$
\mathrm{U}_{0}=\frac{3}{\pi} \int_{0}^{\pi / 3} \sqrt{3} M \sin \left(w t+\frac{\pi}{3}\right) d(w t)=\frac{3 \sqrt{3}}{\pi} M
$$

$\mathrm{M}$ is the $\mathrm{AC}$ side phase voltage amplitude of rectifier. And its value is $1330 \mathrm{~V}$ by calculating. Under the normal operating conditions, the trigger angle of rectifier is zero. The Fourier analysis of the rectified output voltage $U$ can be obtained:

$$
u=2200+125.714 \cos (6 w t)-30.769 \cos (12 w t)+13.622 \cos (18 w t)
$$

In the normal case, two main capacitors are charged by the higher harmonic through the circuit 1$2-3-4$, and the average voltage is $2203.8 \mathrm{~V}$. The voltage is prepared for the deexcitation at all times.

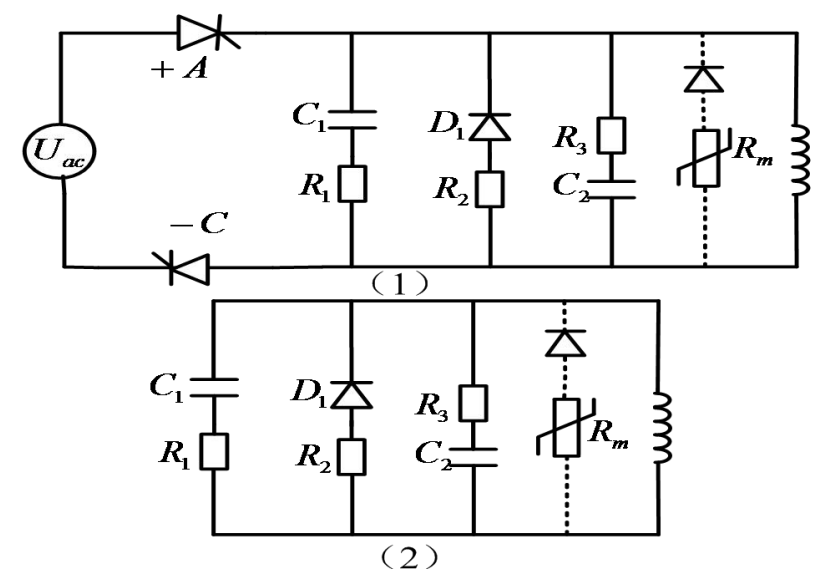

Fig. 2 Diagram of demagnetization process

When the generator starts deexcitation, stop trigger pulse of rectifier and open full-controlled devices both $\mathrm{GTO}_{1}$ and $\mathrm{GTO}_{2}$, then form a circuit model as shown in Fig. 2 (1). The main circuit current of excitation is I, and analysis it through Laplace transform.

$$
I=\frac{U_{a c}-1101.9 / s}{R_{1}+1 / s c_{1}}+\frac{U_{a c}-1101.9 / s}{R_{3}+1 / s c_{2}}+\frac{U_{a c}+13000 L}{s L+r}-\frac{U_{a c}}{R_{2}}
$$

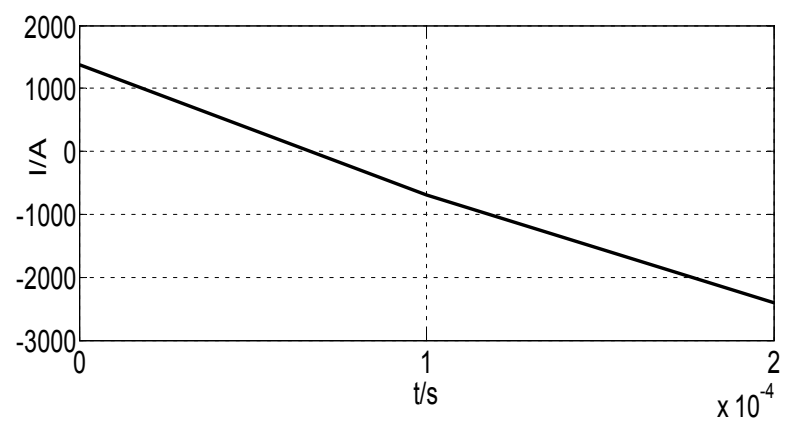

Fig. 3 waveform of current changing

\section{The design of nonlinear resistor for new topology}

When it reach the start conditions of non-linear resistor, the full-controlled devices both $\mathrm{GTO}_{1}$ and $\mathrm{GTO}_{2}$ are closed, which form the circuit structure as shown in Fig. 4(1). After starting the nonlinear resistance $\mathrm{R}$, the energy of excitation winding consume through the nonlinear resistor. Since the energy of the system is concentrated in the field winding, in order to simplify the analysis, the circuit as shown in Fig. 4(2) is used for the energy consumption of non-linear resistor. 

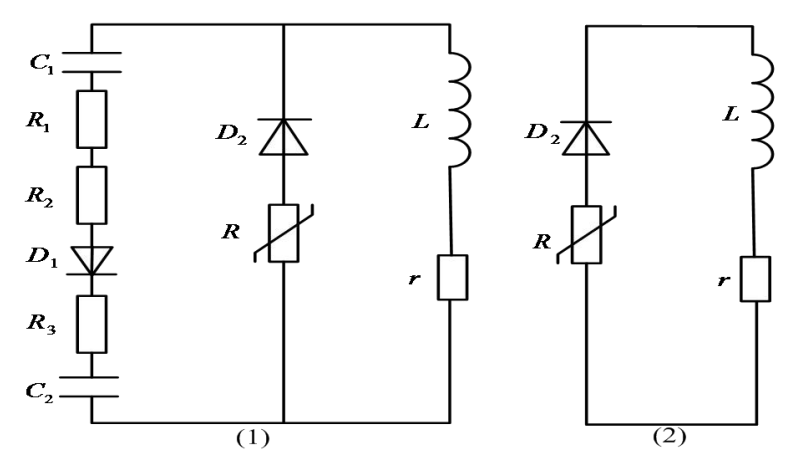

Fig.4 Energy consumption of nonlinear resistor

The voltage characteristics of the non-linear resistor is

$$
I=\left(\frac{U_{R}}{C}\right)^{\alpha}
$$

Where, $\mathrm{I}$ is the current of nonlinear resistor, $\mathrm{A}$; $\mathrm{U}_{\mathrm{R}}$ is the voltage of nonlinear resistor, $\mathrm{V}$; $\mathrm{C}$ is the voltage constant, and related to material of non-linear resistance; $\alpha$ is the nonlinear coefficient.

Obtain from the Fig. 4(2),

$$
L \frac{d i}{d t}+r i+U_{R}=0
$$

Where, $U_{R}$ is the voltage of nonlinear resistor, $V$; $L$ is the inductance of excitation winding, $H$; $i$ is the current of excitation winding, A; $r$ is the resistor of excitation winding, $\Omega$.

Simultaneous the equation both (4) and (5), and apply the Bernoulli equations:

$$
i^{(1-1 / \alpha)}=-\frac{C}{r}+C_{3} e^{-(1-1 / \alpha) \frac{r}{L} t}
$$

Where, $\mathrm{C}_{3}$ is the constant.

The excitation winding current is $\mathrm{I}_{0}$, when the nonlinear resistor is turned on, then

$$
I_{0}=\frac{U_{D}}{r}
$$

Simultaneous the equation both (6) and (7)

$$
C_{3}=\frac{C}{r}+I_{0}^{(1-1 / \alpha)}
$$

Therefore, the equation of excitation winding current $\mathrm{i}$ during the process of generator deexcitation is shown as follows.

$$
i=\left\{-\frac{C}{r}+\left[\frac{C}{r}+I_{0}^{(1-1 / \alpha)}\right] * e^{-(1-1 / \alpha) \frac{r}{L} t}\right\}^{\left(\frac{1}{1-1 / \alpha}\right)}
$$

Since the $1 / \alpha$ is small, and simplify the process, $1-1 / \alpha \approx 1$. When $i=0$, the deexcitation time $t_{m}$ can be obtained

$$
t_{m}=\frac{L}{r} \ln \left(1+\frac{r I_{0}}{C}\right)=\frac{L}{r} \ln \left(1+\frac{U_{D}}{C}\right)
$$

Where $\mathrm{C}$ is calculated as follows

$$
C=Q \frac{m}{n^{1 / \alpha}} C_{0}
$$


Where, $\mathrm{Q}$ is the dispersion coefficient, which ranges from 0.8 to 0.9 ; $\mathrm{m}$ is the number of nonlinear resistors in series; $\mathrm{n}$ is the number of non-linear resistors in parallel; $\mathrm{C}_{0}$ is the voltage constant of Single nonlinear resistor.

Under the conduction of no-load strong excitation in error, $\mathrm{U}_{\mathrm{R}}=2300 \mathrm{~V}$. The dispersion coefficient $\mathrm{Q}$ is 0.85 . The $\mathrm{m}$ is 10 . The $\mathrm{n}$ is 16 . The constant $\mathrm{C}$ change between 200 and 3000 . The non-linear coefficient $\alpha$ is 25 . Then the de-excitation time $t_{m}$ is $0.45 \mathrm{~s}$, and the total time of the de-excitation process is $0.51 \mathrm{~s}$.

\section{The simulation of topology for new deexcitation}

The process of Generators de-excitation is a complex electromechanical process, and the original of the deexcitation system will be need to dealt with idealization. The condition of three times of the rated excitation current act as the condition of no-load strong excitation in error, and the current approximate 13000A. Based on the above analysis, the deexcitation system is simulated by MATLAB.

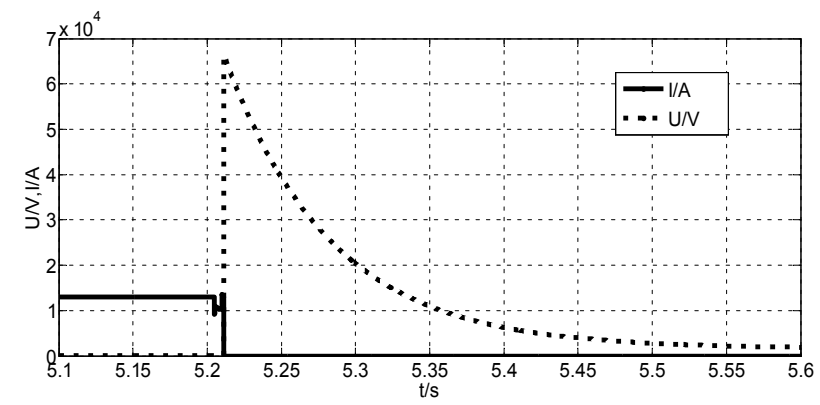

Fig.5 The voltage and current curve of excitation main circuit

The voltage and current curves of the main circuit is shown in Fig. 5. The excitation system of large generation reach the state of strong excitation in error at the time of $5.2 \mathrm{~s}$, and the new softswitching deexcitation system is turn on. Under the function of commutation circuits and the softswitching, the current of excitation main circuit rapidly drops to zero, and the main circuit switch $\mathrm{MK}$ is opened at this time. It is also produce a overvoltage of $6.5 \times 104 \mathrm{~V}$, but the current has been reduced to zero. So the main circuit switch MK don't product arcing in the period, and the main circuit switch MK can be safely opened.

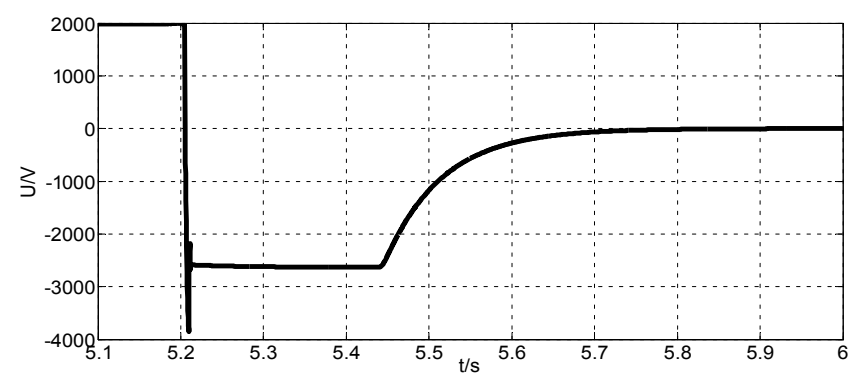

Fig.6 The built of voltage in main capacitor

The building voltage curve of main capacitor is shown in Fig. 6. When the excitation system is working normally, the higher harmonic current from rectifier charges main capacitor. when the deexcitation begins, two main capacitors begin to discharge. After the main circuit current drops to zero, the main circuit switch MK is opened, then the excitation winding reverse charged main capacitor. After the voltage of main capacitors is sufficiently high, and it can start the non-linear, the structure of capacitors connected in parallel is changed into a structure connected in series. Then the non-linear began to consume internal energy. In this process, the circuit structure and parameters should be symmetrical or same, then both current of two capacitors will be approximate, 
but the small differences of both current product a small overvoltage at the same time, and the effect can be ignored.

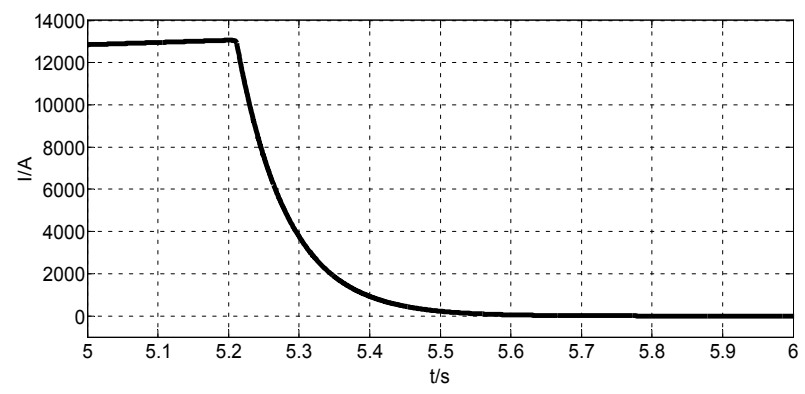

Fig.7 The current waveform of magnetizing equivalent

The current curve of excitation inductance is shown in Fig. 7. It reaches an internal fault state of generator at the time 5.2s. Through the application of the new deexcitation system, excitation equivalent of the inductor current begins to drop, about 5.6 seconds, completely reduced to zero, this deexcitation process lasts about 0.4 seconds to complete.

\section{Conclusions}

According to the parameters characteristic of the "Three Gorges" generator, the configuration parameters of no-load rated condition for ideal accident deexcitation is $\mathrm{T}_{\mathrm{mo}}=0.167 \mathrm{~T}_{\mathrm{do}}$, about 1.8 seconds. The time of short accident deexcitation is $T_{d}^{\prime} / 6$, about 0.6 seconds. $T_{d o}$ is the time constant of field winding, and $\mathrm{T}_{\mathrm{mo}}$ is the deexcitation time of accident. Both are the specific indicators of fast and effective accident deexcitation effectiveness of two typical conditions, and the guidelines of a qualified accident deexcitation. From above theoretical analysis, the deexcitation time of no-load strong excitation in error is $0.51 \mathrm{~s}$, and the simulation experiment result is $0.4 \mathrm{~s}$, which both meet the deexcitation requirement of quick and effective. The design achieve the desired result. Since the circuit model are simplified at theoretical analysis process, its deexcitation time is larger than the simulation results, but both of them meet the above deexcitation evaluation.

\section{Acknowledgement}

This work was financially supported by the National Natural Science Foundation (51577157).

\section{References}

[1] Dake Huang. The difficulties and breakthroughs of large generator internal fault protection technology. The national large and medium-sized hydropower technology collaboration, technology exchange meeting, 2009, No.9, pp.232-237. (in Chinese)

[2] Jianhang Liang, Guanghua Gao, Hongjun Chen, Laiyong Zou. Several major issues of the large generator de-excitation system design. Yangtze River, 2011, No.15, pp.86-91. (in Chinese)

[3] Park,S.H., Cha,G.R., Jung,Y.C., Won,C.Y. Design and Application for PV Generation System Using a Soft-Switching Boost Converter With SARC. Industrial Electronics, IEEE Transactions on, 2010, Vol.57, No.2, pp.515-522.

[4] Ivanovic,B., Stojiljkovic,Z. A novel active soft switching snubber designed for boost converter. Power Electronics, IEEE Transactions on, 2004, Vol.19, No.3, pp.658-665.

[5] Jianhang Zhu, Qinyue Tan, Yuntao Zou,Hua Zhao, juncheng Zhang, Baohui Zhu, Lei Zhou. Improvement of de-excitation system for large generators based on soft-switching technology. Power System Protection and Control, 2014, Vol.42, No.24, pp.116-120. (in Chinese) 